\title{
Template Incision Method
}

National Cancer Institute

\section{Source}

National Cancer Institute. Template Incision Method. NCI Thesaurus. Code C122174.

A method for assessing bleeding time in which two controlled incisions are made on the forearm, using a template to guide placement of the scalpel or blade. 